\title{
procesos de poblamiento coloniales en la nueva granada: el caso de la minería antioqueña (siglo xvii y xviii) ${ }^{\star}$
}

\author{
Juan-Santiago Correa \\ Economista de la Universidad Externado, Magíster en Historia de la Universidad Javeriana, candidato \\ a doctor en Sociología Jurídica e Instituciones Políticas de la Universidad Externado. Director de \\ Investigación del Colegio de Estudios Superiores de Administración -CESA- y Coordinador de la \\ línea en Economía del Grupo de Investigación en Innovación y Gestión Empresarial en el CESA
}

\section{RESUMEN}

El texto analiza los procesos de territorialización hispánica en la provincia de Antioquia (Nueva Granada) durante los siglos XVI y XVII. La hipótesis central gira en torno a la propuesta sobre el papel de los núcleos urbanos como elementos articuladores en la construcción del territorio y como indicadores del dominio efectivo del mismo. Así, se analiza para este período la existencia de una relación multidireccional entre el espacio y la sociedad, en la que ninguno de estos dos elementos es pasivo ni independiente del otro.

Palabras clave: Territorialización, Antioquia, minería colonial, centros urbanos, Colombia

\begin{abstract}
The text analyzes Hispanic territorialization processes in the province of Antioquia (Nueva Granada) during the sixteenth and seventeenth centuries. The central hypothesis revolves around the proposal on the role of urban areas as articulated in the construction elements of the territory and as indicators of effective control of it. Thus, this period is analyzed for the existence of a multidirectional relationship between space and society, in which none of the two components are passive and independent of the other.
\end{abstract}

Key words: territorialización, Antioquia, Colonial mining, urban centers, Colombia

* Submetido: setembro, 2010; aceito: novembro, 2010. 
La explotación aurífera, como eje central de la economía antioqueña, estaba representada en dos sentidos. En primer lugar, el oro se constituyó en el motor de la actividad económica de la provincia y, en segundo lugar, el oro funcionó como moneda para el intercambio y como capital disponible para realizar nuevas inversiones. De esta manera, el metal fungía como mercancía y como medio de pago, constituyéndose como articulador de la provincia al comercio imperial español; por tal razón, el estudio de los ciclos de producción aurífera se ha convertido en el eje central de la mayor parte de los estudios sobre el período colonial antioqueño. ${ }^{1}$

De manera tradicional, se ha afirmado que la explotación minera en Antioquia atravesó dos grandes ciclos estructurales: el primero entre 1550 y 1620 y, el segundo, entre 1680 y 1820. El interregno entre estos dos ciclos para Antioquia ha sido considerado como un período de una profunda crisis económica en el que la provincia apenas pudo sostenerse a sí misma, a pesar del hecho que este período del siglo XVII es testigo de uno de los procesos de territorialización más sólidos, pues se consolidan ejes territoriales sobre los que se mantuvo con éxito una dinámica de circulación mucho más amplia, que tiene como uno de sus centros primarios el valle de Aburrá.

Este fue un proceso en el cual inicialmente Santafé de Antioquia era el principal centro político y económico de la provincia, pero se fue desplazando paulatinamente hacia el Valle de Aburrá, generando una relación asimétrica entre estos dos centros urbanos, en la cual Medellín, en elValle de Aburrá, salía ganando. Esto se dio debido a que Santafé de Antioquia no generaba otros bienes distintos al oro para el comercio intraprovincial mientras que Medellín si lo hacía. ${ }^{2}$

En términos generales, los estudios sobre procesos territoriales en Antioquia no han tenido en cuenta diversas situaciones que podrían dar sentido a esto más allá de la explicación tradicional, en la que se afirma que esta expansión de la frontera productiva hacia el valle de Aburrá y

1 Suárez, Ivonne, Oro y sociedad colonial en Antioquia ,1575-1700. (Medellín: Imprenta Departamental, 1993), 27.

2 Archivo Histórico de Antioquia (en adelante AHA), Colonia, Reales Provisiones, tomo 14, documento 486, ff. 15. 
hacia las tierras altas antioqueñas obedeció a una forma de buscar nuevas alternativas de explotación económica ante una crisis minera en las zonas tradicionales de explotación. Como se verá en este trabajo, la explicación causal entre minería y poblamiento es insuficiente para explicar la dinámica territorial que se estaba presentando en Antioquia a mediados del siglo XVII, en la que si bien la minería seguía constituyendo el motor principal de la economía, era apenas uno de los muchos circuitos económicos que interactuaban, pero con asimetrías en sus tiempos, con diferentes velocidades de interacción y con disímiles niveles de influencia en la dinámica de apropiación del espacio.

Es necesario tener en cuenta que, independientemente de las técnicas y los tipos de minas en explotación, la circulación del oro no se hacía necesariamente por intermedio de los mineros, sino que obedecía a circuitos mucho más amplios, en los que comerciantes y ganaderos jugaban un papel fundamental al registrar el oro en polvo en casas de fundición que no eran necesariamente las mismas en donde se extraía. Este escenario introduce una divergencia en las cifras de producción en las que aparentes caídas de la producción se deben más al lugar de registro que a la producción real. Esta situación será estudiada al inicio de este texto.

Es importante apuntar que la dinámica y la dimensión del negocio ganadero ha sido continuamente subestimada para Antioquia, relacionándola sólo con una pequeña producción concentrada en el valle de Aburrá, orientada al abastecimiento de Santafé de Antioquia; sin considerar que la provincia era un centro importante de este negocio y que sus redes llegaban mucho más allá del simple abastecimiento de un centro minero en decadencia, con lo que la circulación y el registro del oro en polvo, captado por los ganaderos en las zonas mineras, era mucho más amplio de lo que hasta ahora se ha considerado.

Estos circuitos, en su parte final, solían realizar el suministro de carne fresca en los centros urbanos por medio de un contrato o remate de carne. Las autoridades de los diferentes núcleos de población otorgaban este contrato al ganadero que ofreciera al matadero local los precios más bajos por un determinado número de cabezas de ganado. Este tipo de sistema contractual, que fue empleado en la mayoría de los centros mineros y administrativos de la Nueva Granada, permitía un abastecimiento relativamente regular de carne, un pago permanente en tributos 
a los cabildos, y le garantizaba al ganadero que todos los habitantes de la zona estuviesen obligados a comprar su carne. ${ }^{3}$

Sin embargo, es probable que muchas de las estancias cercanas a los centros mineros sacrificaran su propio ganado sin pagar los impuestos de degüello que debían cancelarse en los mataderos, e invirtieran un valor menor al que pagarían en el mercado. Para 1679, esto ocasionaba una pérdida al Cabildo de los dos tomines que debían ser cancelados por cada res que se sacrificara en el valle de Aburrá. ${ }^{4}$

En el mismo sentido, es importante considerar que el fortalecimiento de la arriería a lo largo del siglo XVII, le permitió a los comerciantes controlar una proporción cada vez mayor del oro en polvo, utilizado por muchos mineros y mazamorreros no sólo como mercancía sino como medio de cambio, lo que imprime una distorsión de las cifras de producción, pues los comerciantes no estaban obligados a quintar en la Caja Real donde realizaban el negocio sino que lo podían hacer en cualquier otro sitio donde la tarifa del quinto resultara más favorable o, simplemente en el lugar donde terminara su correría comercial.Aunque el fortalecimiento de las redes comerciales y ganaderas no se analizará en este texto, estas se tendrán en cuenta en la medida en que ayuden a ampliar las explicaciones en torno a la circulación del oro como medio de pago. ${ }^{5}$

Por otra parte, poco se han estudiado los sitios mineros en distintas partes de la provincia que, para el mismo período, se encontraban en producción con resultados importantes. Estos centros mineros, que se analizarán en el tercer apartado, se encuentran precisamente en las zonas de expansión territorial del siglo XVII y, probablemente, se configuraron en el principal motor de dicha expansión, al incorporar nuevas zonas de producción y al ampliar y diversificar los circuitos económicos de la provincia. Estos centros, por su posición geográfica, se orientaron hacia el río Magdalena y no hacia el río Cauca, primer eje de territorialización

\footnotetext{
3 AHA, Colonia, Indios, tomo 24, documento 726, ff. 265r a 267v, 273r.

4 AHA, Colonia, Indios, tomo 24, documento 726, ff. $265 \mathrm{v}$.

5 Williams, Caroline, "Resistance and rebellion on the Spanish frontier: native responses to colonization in the Colombian Choco, 1670-1690," Hispanic American Historical Review, 79.3 (1999):397-424.
} 
de la provincia, generando con esto dinámicas de apropiación del espacio diferentes a las del XVI.

Estos dos ríos, el Cauca y el Magdalena, se constituyeron en los límites naturales de la provincia, de acuerdo con la Real Cédula del 24 de agosto, que se ubicaba entre los ríos Cauca y el Magdalena con el nombre inicial de la gobernación de Entre los dos Ríos, la cual ampliaría más adelante su jurisdicción hasta incluir a Santafé y sus ricos yacimientos de oro. ${ }^{6}$

Los puertos fluviales a lo largo de estos dos ríos eran celosamente controlados por las autoridades, pues el flujo de mercancías permitía el cobro permanente de impuestos. El funcionario oficial que se encargaba de recolectar los derechos de transporte, los fletes y las tarifas era el alcalde de puertos. A partir de 1652 esta concesión oficial cubría los puertos de Cáceres y el Espíritu Santo, sobre el río Cauca; Zaragoza, en el río Nechí; Tenerife, Mompox, Simití, Carare, Nare y Honda sobre el río Magdalena. De estos puertos, los de Zaragoza, el Espíritu Santo y Nare se configuraron como los más importantes lugares de entrada de las mercancías españolas y esclavos destinados a los campos mineros antioqueños. No obstante, el control efectivo era más bien tenue y el contrabando era una situación permanente más que ocasional para la provincia. $^{7}$

Si se tiene en cuenta esta consideración, es posible afirmar que las cifras de contrabando distorsionan los totales fundidos y quintados en la provincia, en especial desde que comenzó la apertura de la frontera chocoana y, con ella, la apertura de las rutas de contrabando por el río San Juan y Atrato. Las permanentes divergencias entre las cifras de producción y arribo a España del oro y que, como se verá en la última parte del texto, no obedecen a rezagos temporales entre una y otra situación, son indicadores importantes del peso del contrabando en la salida del oro en polvo, sin fundir y quintar, como medio de pago. Por supuesto esta situación, permanente a lo largo del siglo XVII, genera serias distorsiones en las cifras sobre las que se ha caracterizado la producción minera antioqueña para este período.

\footnotetext{
6 AHA, República, Estadística y censos, tomo 2689, documento 7, ff. 259r a 261v.

7 AHA, Colonia, Indios, tomo 24, documento 726, ff. $265 \mathrm{r}$.
} 
Así, la minería de oro jugó un papel fundamental en el dinamismo de la economía antioqueña durante la colonia. Desarrollando, en la medida en que se incorporaban nuevas zonas a la frontera minera, el mercado interno de la provincia, fortaleciendo los vínculos con el mercado externo y fomentando el intercambio interregional con otras provincias de la Nueva Granada. La minería antioqueña se realizó mediante la explotación de dos tipos básicos de minas: las de aluvión y las de veta o filón. Bajo la primera denominación, el oro se encontraba en los playones o placeres de los ríos, en donde estaba mezclado con el suelo, encontrándose en forma de partículas o pequeños granos, hasta macizos. ${ }^{8}$

Estas minas eran explotadas por mazamorreros, conocidos así porque el maíz en sus diferentes formas era parte fundamental de su alimentación, quienes requerían pocas herramientas para la explotación minera, entre las que estaban el azadón, el almocafre o azada manual, el barretón para remover material y la batea para separar el oro o la plata de sus gangas o escorias. El azadón, con su hoja de hierro forjado, fue una innovación tecnológica introducida por los españoles, mientras la batea era una pieza cóncava de madera que fue heredada de las técnicas de explotación indígena. Un buen mazamorrero, con estas herramientas, podía procesar entre uno y cinco metros cúbicos de arena o grava en diez o doce horas. ${ }^{9}$

A pesar de que se ha considerado de manera tradicional al oro en polvo como medio de intercambio, hay que tener en cuenta que era el producto de la actividad minera de la región y, por lo tanto, estaba destinado al intercambio con otros bienes. Por tal razón era considerado por los mineros como una mercancía más que como dinero en el estricto sentido. Por tal razón, en la medida en que más rápido lograban cambiar el oro por otras mercancías de consumo, mayor satisfacción obtenían, pues la finalidad del mazamorrero y del pequeño minero era

8 Nisser, Pedro, La Minería en la Nueva Granada (Bogotá: Banco de la República, 1990 (1834)), 17.

9 Brew, Roger, El desarrollo económico de Antioquia desde la Independencia hasta 1920. 2 ed. (Medellín: Universidad de Antioquia, 2000), 50. Jaramillo, Jaime, "La economía del Virreinato (1740-1810)" en José Antonio Ocampo (comp). Historia económica de Colombia. 4 ed. (Bogotá:Tercer Mundo-Fedesarrollo, 1994), 19. Poveda, Gabriel, Dos siglos de historia económica de Antioquia (Medellín: Pro Antioquia, 1979), 15. 
más la subsistencia que la acumulación, llegando incluso a enajenar la producción por medio de las compras a crédito, antes de extraerla. Sin embargo, el cobro de estas deudas no siempre era una tarea fácil y los comerciantes debían recurrir a las autoridades con el fin de recobrar el monto adeudado. ${ }^{10}$

Los mazamorreros, conocidos también como barequeros o zambullidores, eran hombres trashumantes y no tenían concesiones territoriales, es decir, el derecho otorgado por el Rey, a través de sus representantes, para explotar una mina debidamente denunciada, ni poseían cuadrillas de esclavos (generalmente compuestas por cinco esclavos), ni sus herramientas, a excepción de las que contenían hierro, pues eran relativamente baratas. Esta forma de trabajo fue la predominante en el territorio antioqueño. ${ }^{11}$

Las minas de veta o filón se encontraban en zonas rocosas, en donde existía un hilo de oro o plata que penetraba la montaña. Debido a la facilidad y a la abundancia de las minas de aluvión, la explotación de las minas de veta fue muy limitada y se restringía a los lugares en donde había evidencia de haber sido explotada por los indígenas. Una tercera categoría, aunque no tan importante como las dos anteriores, eran los "oros corridos", técnica de explotación muy similar a la minería de aluvión, que consistía en la extracción de los metales que arrastraban los ríos, y que junto con el mazamorreo, constituían cerca del 80 por ciento de la explotación aurífera en Antioquia. ${ }^{12}$

Algunos mineros, con el capital suficiente para poseer una cuadrilla de esclavos, se constituían en empresas que explotaban, generalmente, un territorio de su propiedad. Algunas de estas empresas, formaban unidades administrativas llamadas Reales de Minas, y facilitaban las tareas fiscales de la Corona, al tener ésta más control sobre la producción minera y por lo tanto, sobre la recolección de los impuestos y las tarifas. ${ }^{13}$

\footnotetext{
10 AHA, Colonia, Real Hacienda, tomo 103, documento 2827, ff. 212r

11 Poveda, Dos siglos..., 20.

12 Jaramillo, “La economía...”, 49 y 52. Los “oros corridos' era una explotación aurífera más móvil y, en la que no necesariamente existían reales o concesiones de minas.

13 Jaramillo, "La economía...", 52.
} 
Sin embargo, hay que recalcar que la introducción de las cuadrillas de esclavos se debió a la dificultad de incorporar mano de obra indígena al trabajo minero en Antioquia. La introducción de estos esclavos, aunque se haya constituido en la principal fuente de mano de obra en la minería, representó una inversión, cuyo mantenimiento era alto y costoso; no sólo la compra de los mismos, sino, su manutención se convertía en un costo adicional, que reducía las tasas de rentabilidad de los mineros, convirtiéndose, en el largo plazo y en la medida que los yacimientos se agotaban, en una forma antieconómica de explotación minera. ${ }^{14}$

La minería de aluvión no estuvo sometida a estos costos adicionales, al estar constituida en su mayoría por mineros autónomos e independientes, con una resistencia mayor, debido a sus escasos niveles de capital, a tomar los riesgos que implicaba la inversión en una mina de veta. Estos mineros debían salir constantemente a buscar nuevos yacimientos, cuando la producción de los anteriores disminuía, lo que imprimía una mayor movilidad económica a las zonas de frontera en permanente desplazamiento. ${ }^{15}$

Esta movilidad marca para toda la Nueva Granada un patrón de poblamiento que sigue en buena parte las zonas de vertiente. En el Mapa No. 1, se puede ver que la dinámica de la explotación aurífera se ve acompañada de un patrón de poblamiento similar, en el que los principales yacimientos son también el asentamiento de las ciudades o villas y, en la medida en que estas explotaciones son más ricas, la densidad de los núcleos urbanos aumenta. De manera parecida, estos asentamientos son en su mayoría nodos de comercio que interconectan los mercados regionales, interregionales y el comercio exterior. ${ }^{16}$

Se puede apreciar en el Mapa No. 1, como la minería de aluvión es la predominante en Antioquia y, aunque esta era explotada tanto por mineros como por mazamorreros, la diferencia fundamental entre estos no era la técnica de explotación, pues ambos explotaban de hecho los

14 Uribe, María Teresa y Jesús Álvarez, Raíces del poder regional: el caso antioqueño (Medellín: Universidad de Antioquia, 1988), 5.

15 López, Álvaro, Migración y cambio social en Antioquia durante el siglo XIX. (Bogotá: Centro de Estudios Sobre Desarrollo Económico (CEDE), 1976), 16.

16 AHA, Colonia, Indios, tomo 24, documento 726, ff. 266 r. 
mismos tipos de minas, sino que los últimos utilizaban mano de obra esclava o asalariada, realizaban operaciones previas a las explotaciones mineras más elaboradas, obteniendo ganancias mayores. Estas operaciones previas, que podían tomar de 4 a 6 años, estaban orientadas básicamente a canalizar el agua del río, con el fin de separar las arenas auríferas de otros materiales y más pesadas y, de ésta manera, aumentar la producción de oro.

Esto presentaba varios problemas. En las tierras bajas había demasiada agua, por lo que el minero debía construir trinchos u obstáculos, para disminuir su flujo. En las tierras altas, como Santa Rosa de Osos, el problema era el contrario. El suministro de agua dependía casi exclusivamente de las estaciones lluviosas, y el flujo de la misma no era suficiente para realizar las operaciones en la mina; esto obligaba a los mineros de tierra alta a construir tongas o caídas a desnivel en los cauces de los ríos, con el fin de aumentar la fuerza de la corriente de agua. ${ }^{17}$

La minería antioqueña combinaba, de esta manera, diversas técnicas, tanto indígenas con la batea como hispánicas con la introducción de herramientas de hierro, que dependiendo del tipo y el lugar de explotación requerían diversos niveles de inversión de capital. Además, al predominar la explotación de minas de aluvión, la frontera minera, por su carácter móvil, incorporaba, de forma relativamente fácil, nuevas zonas a los territorios ya consolidados, las que una vez integradas continuaban funcionando en la dinámica territorial de la provincia.

Por otra parte, es importante tener en cuenta que a partir de las ordenanzas de Rodas, en 1584, se preveía que cuando se descubría una mina, el señor de cuadrilla no podía despedir a los mineros a sueldo que tenía contratados mientras estuviera en producción o de lo contrario debía pagarles el salario durante un período de tiempo igual. Sin embargo, el minero que abandonara la cuadrilla no podía servir a otro en el término de dos años. Además, el minero a sueldo tenía prohibido adquirir el derecho sobre minas, aun teniendo una cuadrilla su cargo.

Estas ordenanzas generaban fácilmente un sinnúmero de conflictos sobre la propiedad de las minas, el derecho a explotarlas y, la mutua dependencia entre señores de cuadrilla y mineros a sueldo. Sin embar-

17 Twinam, Ann, Mineros, comerciantes y labradores: Las raíces del espíritu empresarial en Antioquia, 1763-1810 (Medellín: FAES, 1985), 81 y 82. 
go, dirimir estos conflictos no era una tarea fácil en una zona de frontera minera y el descubrimiento de una nueva mina era una tentación permanente para que cada uno se estableciera por su cuenta, o mantuviera en secreto durante un tiempo la existencia de la misma. Esta situación imprimía sobre las minas que no eran denunciadas, por una u otra razón, un nivel de inseguridad y desprotección legal amplio, que no sólo le dieron una mayor movilidad a la frontera productiva, sino que agravó de manera significativa la fiabilidad de las cifras de producción. ${ }^{18}$

En un sentido similar, se ha sostenido de forma tradicional que la economía minera en la Nueva Granada y en Antioquia atravesó dos grandes ciclos de explotación aurífera. Como se puede apreciar en la gráfica No. 1, existe un primer momento que no se puede clasificar estrictamente como ciclo minero, pues no se hace minería, sino que se procede a una apropiación de los tesoros indígenas por parte de los conquistadores españoles. Después del primer momento, empieza un primer ciclo, circunscrito principalmente al Nuevo Reino (Santafé de Bogotá, Tunja,Vélez y Pamplona), a la gobernación de Popayán y a la provincia de Antioquia, comprendido entre los años de 1550 y 1620.

Este ciclo se caracterizó por la explotación de lavaderos fluviales o aluviones, mediante la utilización de trabajo indígena; el posterior decaimiento de la producción se debió al agotamiento de la tecnología, y al tipo de explotación, propias del primer ciclo del oro, cuya decadencia empieza a ser muy marcada a mediados del siglo XVII. ${ }^{19}$

\footnotetext{
18 Colmenares, Germán, Historia económica y social de Colombia, 1537-1719. 5 ed. (Bogotá: Tercer Mundo, 1997), 293-294

19 Colmenares, Historia económica..., 328.
} 
Mapa No. 1. Actividad minera durante la colonia.

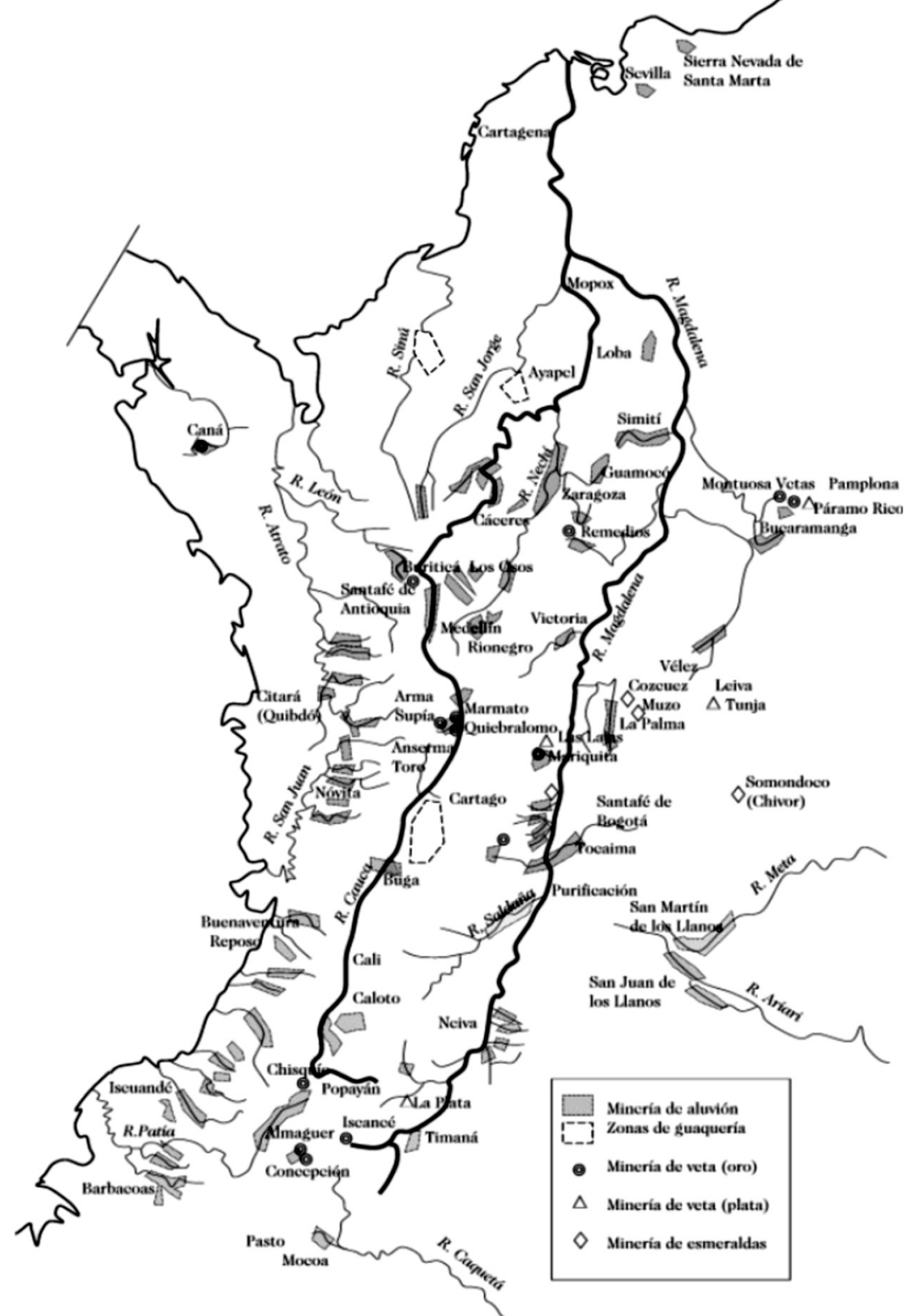

Fuente: West, Robert C. Colonial placer mining in Colombia (Baton Rouge: Louisiana State University Press, 1952), 10 (modificado por el autor) 
El segundo ciclo, entre 1680 y 1820, tuvo como origen el descenso demográfico de la población indígena; este período se centró fundamentalmente en el Chocó y en la ampliación de la frontera minera en Antioquia, caracterizándose por la explotación de la minería de veta y por la utilización de la mano de obra esclava. Sin embargo, estos ciclos estuvieron ligados más que al tipo de mina que se explotaba, de lo que a la expansión de la frontera de explotación. ${ }^{20}$

Grafica No. 1. Producción aurífera registrada (miles de pesos plata)

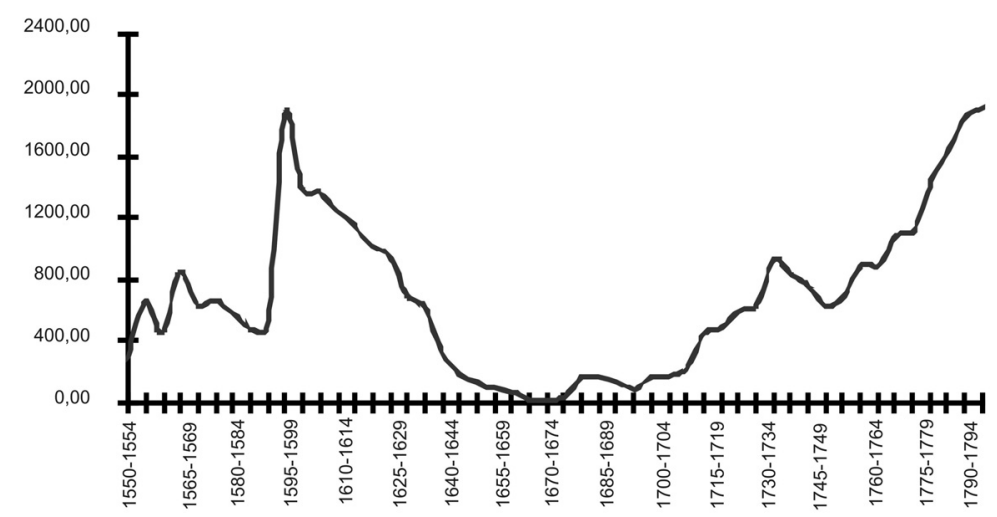

Fuente: Jaramillo, “La economía...," 51

En la gráfica No. 1, construida con cifras tomadas de los registros oficiales de la Cajas Reales, se puede observar como tras un auge bastante significativo en las primeras décadas de explotación sobreviene la crisis del primer ciclo, la cual se puede apreciar en las gráficas No. 1 y 2 por la drástica disminución de la producción. Esta caída se origina en el agotamiento de las técnicas indígenas, ya que los "oros corridos" de más fácil extracción habían sido agotados y se requería un tipo de extracción más elaborada. Sin embargo, las innovaciones españolas en herramientas, las cuales facilitarían este tipo de extracción, no se utilizaron extensivamente, pues la mayoría de estas herramientas -aun-

20 Colmenares, Germán, "La formación de la economía colonial (1500-1740)” en: José A. Ocampo (comp). Historia económica de Colombia. 4 ed. (Bogotá.: Tercer MundoFedesarrollo, 1994), 35. Colmenares, Historia económica..., 267 y 328. 
que efectivas- requerían de hierro, y su altísimo costo se convirtió en un serio limitante para proveer a las cuadrillas de trabajadores. ${ }^{21}$

Gráfica No. 2. Primer ciclo aurífero en Antioquia

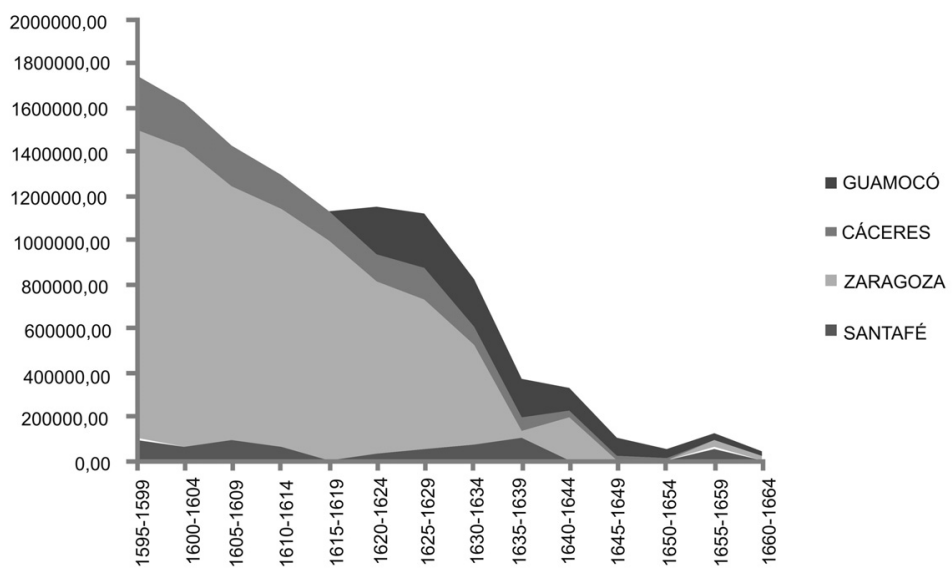

Fuente: Colmenares, Historia económica..., 331.

La información de la gráfica No. 1, sobre la que se sostiene esta caracterización para Antioquia, se obtiene en los totales de oros, fundidos y quintados, provenientes de la producción de oro de Santafé de Antioquia, con sus ricos yacimientos de aluvión en el Cauca y de veta en el cerro de Buriticá; en Cáceres en el bajo Cauca; Zaragoza en el bajo Nechí; y las minas de oro de San Francisco de Guamocó. En este sentido, siguiendo a Colmenares, la producción de oro en Antioquia estuvo marcada por el descubrimiento de nuevos yacimientos y, en poblaciones como Remedios y Zaragoza, estuvo determinada por la cantidad de mano de obra (indígena y esclava) a la que se tuvo acceso, principalmente por las grandes cuadrillas de esclavos en la década de 1590, que hicieron posible un mayor grado de explotación de estas minas. ${ }^{22}$

21 Colmenares, "la formación...", 35.

22 Colmenares, Historia económica..., 328. 
En particular, la importancia de la mina en el cerro de Buriticá no puede ser descuidada, pues no sólo entró en producción muy temprano en la colonia y se constituyó en una de las pocas minas de veta en producción, sino que también registra producción para finales del siglo XVII y para el comienzo de la vida republicana.Además, es de notar que para el siglo XVIII se constituyó en Santafé de Antioquia la sociedad empresarial más temprana de la que tal vez se tenga noticia. Esta sociedad fue constituida por diez socios que aportaron capital en dinero, herramientas y esclavos con el fin de explotar dicha mina y, además de tener estatutos bastante avanzados para la época, definía la forma de distribuir ganancias o asumir pérdidas de forma proporcional al capital invertido por sus socios, el ingreso y retiro de socios y las donaciones religiosas que se quisieran realizar. Este es tal vez uno de los ejemplos más tempranos de una sociedad de negocios del tipo capitalista del que se tenga noticia para la provincia. ${ }^{23}$

En todo caso, el potencial de explotación de estos yacimientos tempranos decayó a lo largo del siglo XVI y XVII, pues la mano de obra indígena fue drásticamente diezmada por diversos factores. Esta, al haberse constituido en el principal elemento de explotación y al no ser reemplazada adecuadamente por la mano de obra mestiza y negra, trajo como consecuencia la disminución en la explotación del oro y coadyuvó a lo que se ha denominado como una crisis estructural del primer ciclo, en la que, los principales centros mineros, como Cáceres y Zaragoza, entraron en un período de decadencia del que no se recuperaron. La producción en Santa Fe de Antioquia había caído, en 1625, a una tercera parte y, en Cáceres y en Zaragoza, llegaba apenas a la mitad de la producción del siglo XVI, sosteniéndose que solamente la extracción de oro en San Francisco de la Antigua de Guamocó permitió atenuar los efectos de la reducción en la producción. ${ }^{24}$

23 AHA, Escribanos, Juan José Lotero, ff. 56v-57.

24 Poveda, Gabriel, 1988. "Breve historia de la minería" en Jorge Orlando Melo (Dir). Historia de Antioquia. (Medellín: Ed. Folio, 1988), 211. McFarlane, Anthony, Colombia antes de la Independencia: Economía, sociedad y política bajo el régimen Borbón. (Bogotá: Banco de la República-El Ancora, 1997), 126. Álvarez,Víctor, "La sociedad colonial, 1580-1720" en Jorge Orlando Melo (Dir). Historia de Antioquia. (Medellín: Ed. Folio, 1988), 55. López, Migración..., 27 y 28. 
En San Francisco, la Antigua de Guamocó funcionó una Caja Real que, por sus registros de oro fundido, llegó a ser considerada como el segundo productor de oro de la Nueva Granada. Como se puede apreciar en la gráfica No. 2, este emplazamiento tuvo un momento de auge significativo entre 1620 y 1630 , aunque comienzan a descender sus registros hasta 1675 , fecha en la que prácticamente había sido abandonada por sus vecinos. ${ }^{25}$

Estos elementos han servido para sostener que, una vez establecido por Rodas el triángulo minero temprano -Santafé, Zaragoza y Cáceres- y la producción de Guamocó, el patrón de poblamiento y territorialización en Antioquia giró alrededor de sus ciclos económicos y auríferos y cualquier posible expansión de la frontera interna sólo se debió a una estrecha correlación con la producción aurífera.

Un esquema de este tipo, centrado en algunos sitios de explotación minera, resulta insuficiente para explicar los procesos de territorialización del siglo XVII, que si bien estuvieron marcados por la producción de oro como elemento dinamizador del mercado, aunque en zonas diferentes a las mencionadas, requiere de un estudio más cuidadoso de los circuitos comerciales y auríferos no sólo al interior de la provincia, sino con los principales centros económicos de la Nueva Granada.

En este sentido, la fundición del oro en polvo se podía realizar en diversos momentos, aunque en todo caso el hecho de ser fundido precisaba la entrada del oro al mundo de la circulación de mercancías y, de esta manera, a diversos procesos económicos en los que se hallaba su poseedor. Si se tienen en cuenta sólo las cifras del oro de fundición, es claro que para el siglo XVII los centros mineros mencionados se encontraban en decadencia; sin embargo, es importante resaltar que el control del oro, de acuerdo al poseedor del mismo, marca momentos y sitios diferentes de registro.

En las reclamaciones sobre las tierras pertenecientes al difunto marido de María Ordaz, Fernando del Toro Zapata, se menciona que en sus minas de los Osos, Guarne, Ovejas y la Onda, se extrajo 'muchas cantidades de oro en aumento de los quintos reales de Remedios..., de lo que se puede inferir que esta situación no era extraña en esta zona.

25 Suárez, Oro y sociedad..., 28. 
En este sentido, para 1575 los comerciantes antioqueños fundían tan sólo el 9\% del oro de la provincia, mientras que para finales del siglo XVII llevaban a fundición el 59\% del oro en polvo, lo que sumado al hecho que el comerciante no tenía restricciones para el sitio donde fundía el oro, se presenta una distorsión significativa de los registros de producción. En el mismo sentido, en las últimas décadas del siglo XVI los ganaderos en Antioquia llevaban a fundición el 10\% del oro en polvo de la Provincia y, para finales del siglo XVII el 33\% del total de la producción registrada. ${ }^{26}$

Mapa No. 2. Principales centros mineros en el siglo XVI

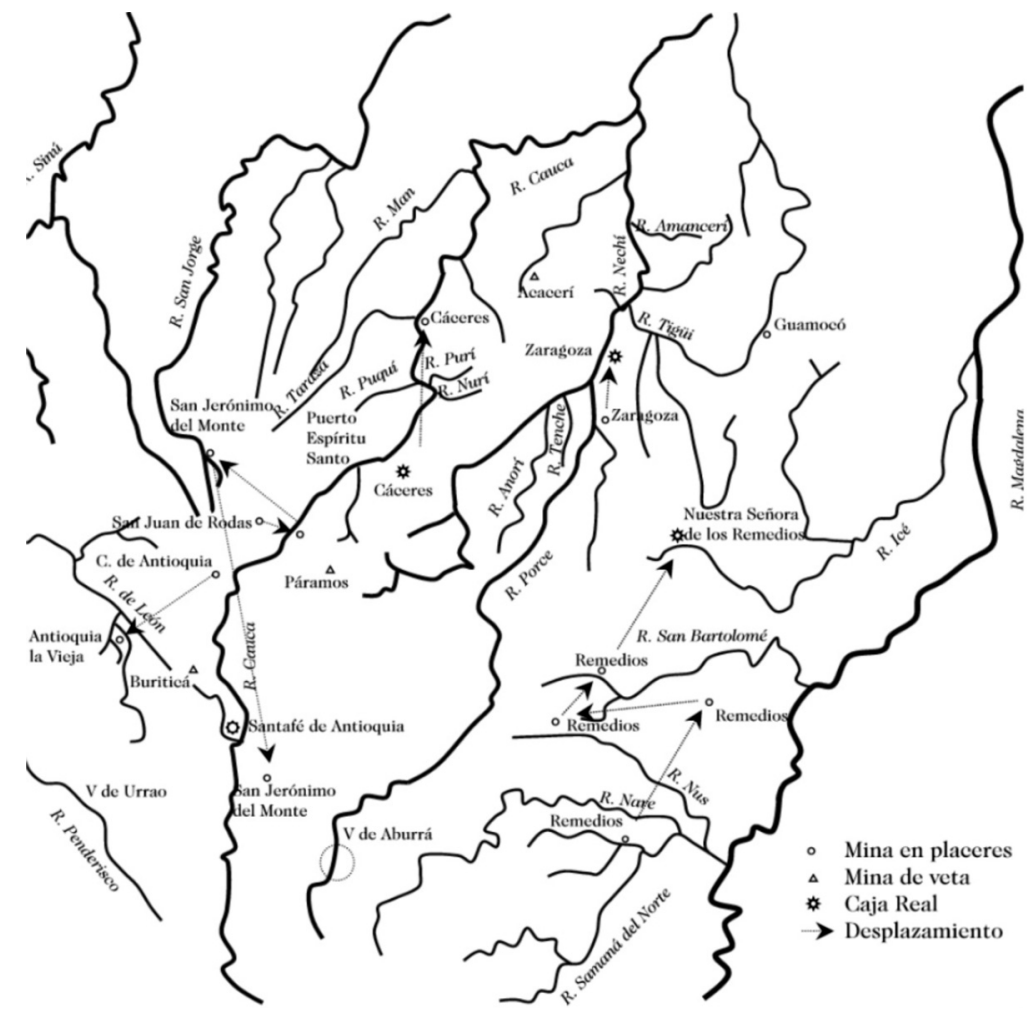

Fuente:West, Colonial..., 24-25 (modificado por el autor)

26 AHA, Colonia, Tierras, tomo 189, documento 4728, ff. 2 r. 
Esta situación implica que el control del oro en polvo a lo largo del siglo XVII dejó de estar bajo el control exclusivo de la actividad minera para concentrarse en las actividades comerciales y ganaderas de la provincia. Es importante resaltar que esto no significa que el oro fuera controlado por diversas personas diferentes, pues lo que se encuentra en la colonia es un empresariado diversificado que actuaba en diferentes esferas económicas con el fin de reducir el riesgo, sino que los circuitos de circulación del oro como mercancía y como medio de pago eran mucho más amplios que el lugar de la producción del mismo.

Por esta razón, el oro que se producía en un lugar no necesariamente era quintado y registrado en la Caja Real del sitio, sino que, dependiendo del circuito comercial o ganadero en el que entraba, podía aparecer registrado en lugares diferentes. Resulta entonces dificil sostener que toda la provincia se encontraba en medio de una crisis minera para este período, pues hay factores que no se han considerado en las cifras y resulta necesario ampliar las investigaciones con respecto a las producciones registradas de cada uno de los centros mineros y rastrear la circulación del oro como medio de pago a través de los circuitos comerciales.

Como se mencionó, las cifras en las que se sustentan los ciclos mineros antioqueños no tienen en cuenta todos los centros mineros de la zona. Este es el caso del último establecimiento de la ciudad de Remedios, trasladada por mineros atraídos por las explotaciones en Zaragoza y por la riqueza de los aluviones del río Nechí, quienes fueron desplazándose continuamente la ciudad, primero a la comarca del río Nus y luego, en 1590, a su emplazamiento definitivo.

En este último lugar, los vecinos de Remedios hallaron los yacimientos de Lomarrica que explotaron con mano de obra indígena y, más tarde, continuaron la explotación, con mano de obra esclava de algunas quebradas afluentes del Nechí (Cana, Nitiniti, Pocoro, Perimana y Niyaba), que Zaragoza reclamaba como de su jurisdicción. La apropiación de terrenos cercanos a esta última ciudad se explica por dos posibles razones: por una parte se ha sostenido que la desidia de los habitantes de Zaragoza y su incapacidad para realizar nuevas poblaciones que, como Niyaba, tan sólo distaban dos leguas o incluso el asiento de Remedios que parece que pertenecía a su jurisdicción. Por otra parte, una explicación más plausible parece ser la poca necesidad que tenían los vecinos 
de Zaragoza de realizar una expansión territorial que distrajera los recursos vitales para la explotación de sus ricos yacimientos auríferos.

No obstante, a pesar de no contar con los recursos suficientes para esta apropiación territorial, los habitantes de Zaragoza tomaron acciones legales -que indudablemente significaban una inversión mucho menor- frente al nuevo emplazamiento de Remedios y en especial frente a sus pretensiones de explotar el oro del Nechí. Realizaron las reclamaciones legales sobre la base que se trataban de regiones comarcanas de los indios encomendados a los vecinos de Zaragoza, aunque no hubiera explotaciones agrícolas directas por parte de ellos. Hacia 1604 el yerno de Rodas, Bartolomé de Alarcón, amenazó con un bloqueo a los suministros de Remedios que explotaban los afluentes del Nechí y los obligó mediante esta acción a quintar y fundir el oro en la Caja Zaragoza. ${ }^{27}$

A pesar de esto, las cifras de Zaragoza disminuyen en estos años, lo que hace pensar que los vecinos de Remedios, con lazos más cercanos a Santafé de Bogotá que a las ciudades y villas antioqueñas, sacaran buena parte del oro en polvo hacia esta Caja Real y prefirieran no fundirlo en Zaragoza o en la distante Santafé de Antioquia. Sin embargo, como se verá más adelante, parte de este oro en polvo circulaba en las rutas de abastecimiento del valle de Aburrá y probablemente tenía un radio de circulación más amplio que el de la Caja de Zaragoza, razones por las que es factible pensar que las cifras se encuentran subestimadas y que, si bien el oro fundido estaba en declive, el oro en polvo, como medio de pago, seguía fluyendo en los circuitos comerciales.

Como en el caso de Remedios, las cifras correspondientes a la producción aurífera de Santa Rosa, Los Osos, San Pedro, Petacas, Guarne y Rionegro, entre otros, han sido seriamente descuidadas en los estudios sobre la minería en Antioquia. Esta zona minera comenzó a ser explotada alrededor de 1634, cuando la frontera de explotación tradicional se desplazó, por su descenso en la producción, a las zonas altas antioqueñas y, para 1644, se otorgaban con frecuencia capitulaciones para el descubrimiento de minas en esta zona. ${ }^{28}$

27 Colmenares, Historia económica..., 278.

28 Parsons, James, La colonización antioqueña en el occidente de Colombia. 4 ed. (Bogotá: Banco de la República-El Ancora, 1997), 78. 


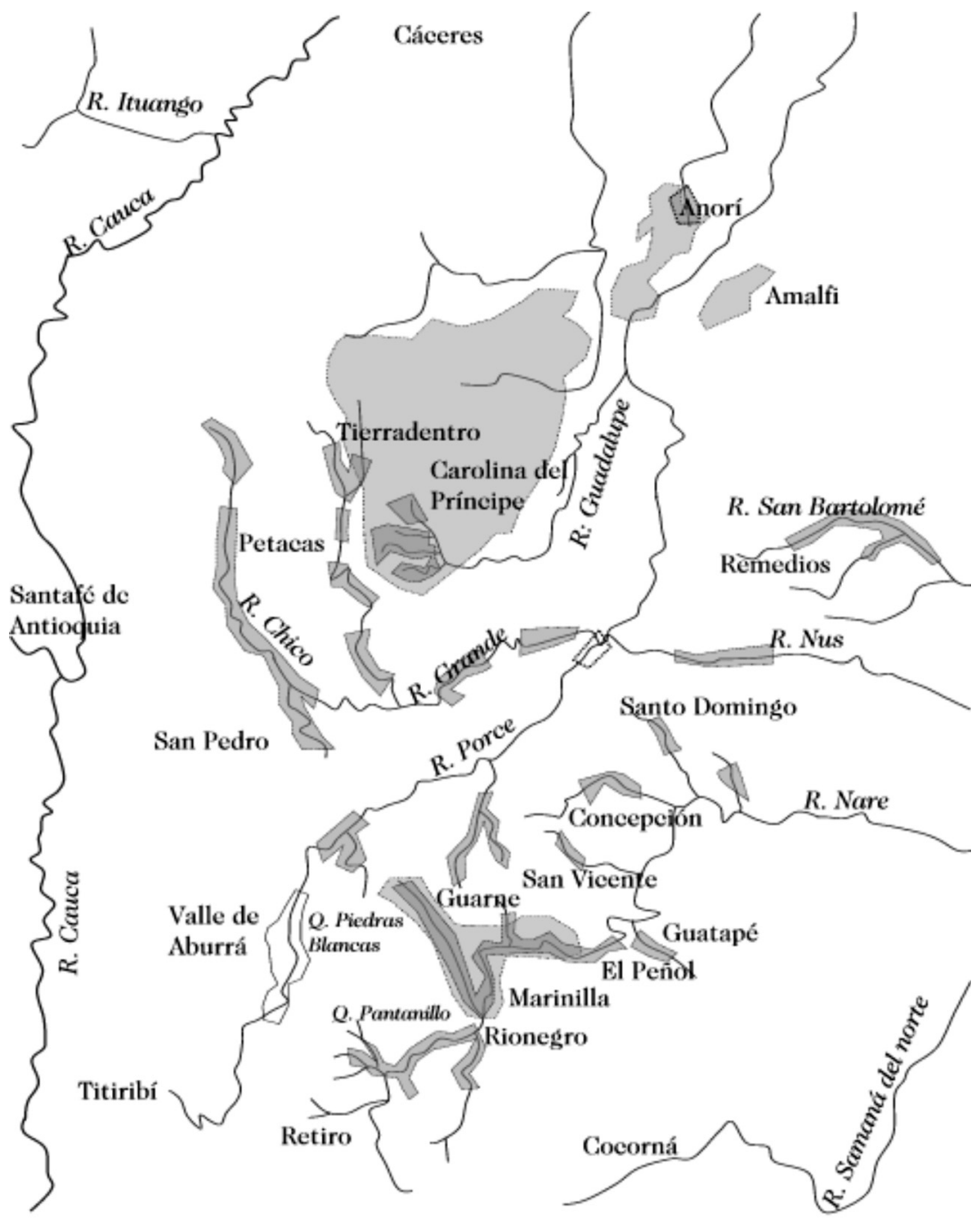

Gravillas auríferas $\quad$ Principales placeres de oro

Fuente:West, Colonial..., 28 (modificado por el autor). 
Se tiene evidencia de minas en explotación en la zona de Guarne al menos desde finales desde el siglo XVI y principios del siglo XVII por parte de mineros que trasladaron su producción, o parte de ella, de las minas del cerro de Buriticá en dicha zona. ${ }^{29}$ Una revisión en el Archivo Histórico de Antioquia en busca de registros de minas en estas zonas, arroja resultados interesantes, pues además de las minas de veta y aluvión pertenecientes a Fernando de Toro, mencionadas anteriormente, se encuentran las de Francisco y Pedro Barbán de Angulo provistas por Gaspar de Rodas, el 7 de septiembre de 1598. Estas minas se encuentran en producción para 1668, cuando se entabla un pleito entre los herederos de estos y Catalina de Ureña por invadir sus predios y explotar ilegalmente estas minas. Este tipo de datos, acompañados de la enorme cantidad de registros de títulos que se encuentran en el fondo Santa Rosa y en los testamentos que reposan en el fondo de Mortuorias, de la sección Colonia del AHA, sugiere que es necesario una revaloración de la información de la producción minera para los siglos XVI y XVII en esta zona aurífera. ${ }^{30}$

Las gravillas auríferas ubicadas en los cerros de las tierras altas antioqueñas ofrecían alrededor de Rionegro una capa relativamente superficial y delgada, fácil para su explotación. En el mismo sentido, cerca de Santa Rosa y Los Osos, estas gravillas ricas en oro se ubicaban a lo largo de extensas áreas que no presentaban mayores dificultades para su extracción. En términos generales, para este período, la gran mayoría de afluentes fluviales de la zona se veían favorecidos en su contenido de oro y no requerían técnicas avanzadas para explotación, lo que fomentó el traslado de muchas de las cuadrillas mineras de las zonas bajas antioqueñas, en especial la de Cáceres y la de Zaragoza, en busca de mejores alternativas de explotación.

El registro de la Caja Real de Santafé de Antioquia muestra que entre 1623 y 1624, se realizaron las primeras explotaciones en Rionegro y que, para 1625, Guarne y la quebrada de La Mosca ya estaban en producción. Además, para principios de la década siguiente, algunos de los mineros antioqueños habían comenzado a remontar el río Porce,

29 AHA, Colonia, Mortuorias, tomo 246, ff. 187-189 (documento 5441).

30 AHA, Tomo 183, documento 4610, ff. $1 \mathrm{r}$ a 2r; documento 4611, ff. $3 \mathrm{r}$ a 6 r; tomo 189 , documento 4728, ff. 1r a 5r; y, Minas, tomo 110, Sección Santa Rosa, tomos 1, 2 y 3. 
desde el valle de Aburrá, con el fin de descubrir y poner en producción placeres en las partes altas de la cordillera. ${ }^{31}$

Siguiendo a West, hacia 1636 había ya solicitudes de permisos a las autoridades legales para explotar minas cerca del río de Los Osos, cerca a la actual Santa Rosa, aunque su explotación comenzó alrededor de 1638, cuando los placeres a lo largo de Riochico, en Los Osos, estaban en producción. Para mediados del siglo XVII los placeres a lo largo de Riochico, Riogrande y, posiblemente, el alto Guadalupe, habían sido reclamados y su minería estaba en operación; en el mismo sentido, la Caja Real de Petacas, la actual Belmira, se encontraba establecida para este período, desafortunadamente los registros de esta caja poco han sido tenidos en cuenta y requieren mayor profundización en los estudios sobre la minería de esta zona aurífera.

La explotación de los minerales se hizo principalmente desde Santafé de Antioquia, cuyos vecinos comenzaron a desplazar los nuevos frentes de explotación minera hacia el valle de Aburrá en 1575, el río Cauca en 1621, la zona de Ovejas, San Pedro y Los Osos entre 1638 y 1644 y, Petacas y Riochico entre 1665 y 1668, dependiendo de la reducción en la producción en Santafé. Esto implica que aunque la producción se reducía en un sitio, los mineros poseían los medios para realizar la apertura de nuevos frentes mineros. ${ }^{32}$

Es importante anotar que si bien la explotación del valle de Aburrá fue bastante limitada y nunca llegó a ser considerada como un centro minero, si se constituyó en un puente para llegar a otras zonas, como las explotaciones en Guarne y La Mosca, en actividad desde 1644, y de allí hacia Rionegro en 1650, Santuario en 1661 y Piedras Blancas en 1668. Hacia el oriente del valle de Aburrá se encuentran en producción para este período las cuadrillas de Diego Álvarez del Pino; en la quebrada Pantanillo la de Facundo Ramírez de Herrera; en varias minas de Rionegro las de Rodrigo García Hidalgo; en Piedras Blancas las de Felipe de Herrera; en las quebradas La Honda, Brizuela y La Jacinta las de Cristóbal de la Parra,; y en La Mosca las de Ana María de Toro Zapata.

\footnotetext{
31 West, Colonial..., 27 a 29.

32 Suárez, Oro y sociedad..., 150.
} 
En el mismo sentido, los vecinos de Santafé entre 1650 y 1675 ampliaron la explotación aurífera más allá de Ovejas y San Pedro. Desde esta zona minera partieron las cuadrillas de Juan García de Ordaz, Lorenzo Cortés de Ordaz y Figueroa, Miguel Martínez de Vivanco, Laureano de Piedrahita, Pedro de la Serna Palacio, María Paladines, Ana María de Toro Zapata y Juan de Toro, hacia Concepción en 1661, Santo Domingo en 1676 y el río Porce en 1675. En el año de 1682, partiendo de Los Osos, la cuadrilla de Cristóbal Ruiz de la Parra amplió el radio de explotación a la zona de Batea Seca.

Un patrón similar de ampliación de la frontera minera ocurrió hacia la zona de Petacas y Riochico entre 1665 y 1668, siendo explotadas sus minas por Fernando de Montoya y Miguel Martínez de Vivanco; Sopetrán y San Jerónimo entre 1665 y 1671; y las minas localizadas entre Urrao y Frontino fueron explotadas por las cuadrillas de Felipe de Herrera y Miguel Martínez de Vivanco. Estas zonas de frontera a su vez sirvieron de punto de partida para nuevas ampliaciones; así en los últimos veinticinco años del siglo XVII se incorporaron Riogrande y Hojas Anchas (1685-1690), en donde se encontraban las cuadrillas de Felipe de Herrera y Miguel Martínez de Vivanco. Entre 1676 y 1700, siguiendo posiblemente la ruta del río Penderisco, se abrieron las explotaciones del Chocó, en donde participaron el gobernador Juan Bueso deValdés, Antonio de Guzmán y Céspedes y Luis de Acevedo y Redes. Los últimos frentes mineros que se abrieron en el siglo XVII, entre 1686 y 1690, fueron La Mina, Anzá y Paramillo, con las explotaciones realizadas por Mateo de Castrillón, Felipe de Herrera y Nicolás de Landeta. ${ }^{33}$

Para principios del siglo XVIII estos centros mineros se encontraban en plena producción. En el área de Rionegro las viejas terrazas de producción que bordeaban a la quebrada de La Mosca, cerca del real de Guarne, eran consideras como unas de las más productivas de Antioquia; aunque muchos campos diferentes a los de La Mosca prevalecían a lo largo del río Ovejos (alto Nare), desde La Ceja hasta Marinilla. Los campos mineros de San Vicente y Concepción, al nororiente de Guarne, tenían también para este período una producción significativa. Al

33 Suárez, Oro y sociedad..., 150 a 152. 
norte del río Porce se habían establecido para este mismo momento los reales de Santa Rosa, Petacas, San Pedro, Don Matías y una serie de pequeños campos en el alto Guadalupe. ${ }^{34}$

Lo que se puede apreciar es que, a lo largo de todo el siglo XVII, la frontera minera se amplió hacia el norte y el oriente de Antioquia. Esta situación obliga a replantear las caracterizaciones realizadas alrededor de la economía minera antioqueña, pues tradicionalmente se han considerado algunos centros mineros, de cuya importancia no cabe duda, pero que no bastan para realizar generalizaciones para toda la provincia y, en lugar de plantearse dos grandes ciclos mineros, posiblemente se encuentre mayor evidencia que permita replantear los totales de producción y se precisen ciclos e interciclos mineros en diferentes puntos de extracción para el siglo XVII, que ayuden a entender de mejor forma los procesos de territorialización y de circulación de hombres y mercancías, no sólo del valle de Aburrá sino de toda la zona.

Otro problema adicional que distorsiona las cifras es el contrabando. No sólo la circulación del oro, con su impacto sobre la propiedad y registro de los totales del oro, así como la presencia de centros mineros alternativos introducen desviaciones que hacen necesario revaluar las cifras de producción aurífera, sino que estas cifras se encuentran subvaloradas debido a la fuga del oro de los circuitos legales durante la colonia.

El contrabando no fue un hecho aislado en la colonia sino una práctica habitual que utilizaron las potencias rivales de España para minar el comercio imperial. Aunque por algún tiempo el imperio español gozó de un acceso privilegiado a los enclaves que los navegantes portugueses habían establecido en África, la separación de Portugal, en 1640, dejó a la Corona sujeta a otras potencias europeas para el vital suministro de esclavos a sus colonias americanas. Además, la ocupación por parte de Inglaterra, Francia y Holanda de Jamaica y algunas de las Antillas menores ocasionó un golpe severo al monopolio comercial que había sostenido España. ${ }^{35}$

Para este mismo período, se estaban haciendo los primeros esfuerzos serios para la apertura de la frontera chocoana y, aunque esta expansión

\footnotetext{
34 West, Colonial..., 30.

35 Colmenares, "La formación...”, 38.
} 
territorial abrió las posibilidades de una explotación minera de consideración, también abrió las rutas del contrabando por los ríos San Juan y Atrato. Los privilegios de trata otorgados a las potencias rivales no sólo ponían al imperio en una situación de dependencia en un elemento vital para la economía colonial, sino que debilitaban el monopolio comercial al favorecer el comercio por fuera de los circuitos legales.

Los navíos extranjeros con privilegio de trata -de permisión - traían los esclavos al puerto de Cartagena y, además, la flota de la carrera de Indias, autorizada a abastecer el comercio americano, con frecuencia se encontraba a su arribo con los puertos copados con mercancías de contrabando. Este tipo de comercio representaba una fuga permanente de oro en polvo, pues el oro al ser utilizado como medio de pago salía del circuito legal sin ser fundido y sin pagar los impuestos y tarifas requeridas.

Por otra parte, el contrabando no sólo se incrementaba por el otorgamiento de grandes licencias y asientos, o contratos de importación masiva de esclavos a cambio del pago de derechos por cada esclavo introducido, sino por el arribo de grandes cantidades de navíos sueltos que procedían directamente de las costas africanas. Una parte importante de estos navíos no poseían licencias o derechos asentistas o sólo justificaban parte de ellas. Sin embargo, dado el inmenso tamaño del negocio, era habitual que las autoridades portuarias no fueran muy estrictas con el cumplimiento de la ley.

A pesar de los esfuerzos realizados por la Audiencia, desde 1589, para verificar, en los lugares de explotación, la legalidad de las licencias, era frecuente que los mineros reportaran sólo parte de sus esclavos, mientras ocultaban a muchos de ellos y encubrían a otros durante las averiguaciones. Además, muchas de las licencias presentadas no eran auténticas o correspondían a esclavos que ya habían fallecido.

A partir de 1590 se enviaron a Cartagena un grupo de funcionarios con el encargo de investigar el contrabando de negros en el puerto. El fiscalVillagómez y el doctor Téllez de Erazo, en 1594; en 1595, Francisco Méndez de Puebla; en 1619, el licenciado Espino de Cáceres; y en 1620 y 1621, el visitador Diego de Medina y Rosales y el licenciado Fernando de Sarria. Para 1641, el oidor Bernardino de Prado Guevara aún averiguaba sobre los fraudes ocurridos en Cartagena desde 1622. Las denuncias generales giraban alrededor de los mismos ilícitos: navíos 
sin licencias, complicidad de los funcionarios, e intereses creados entre los moradores de los puertos ${ }^{36}$.

Probablemente el momento pico en la introducción de mano de obra esclava a Antioquia se encuentra alrededor de 1598, cuando el presidente Sande escribía que en Zaragoza trabajaban cerca de 3.000 esclavos y que para toda la Provincia había unos 6.000 esclavos en labores. Para 1610 se calculaban que en Zaragoza existían cerca de 200 esclavos amotinados que, según Bartolomé de Alarcón, recorrían el triángulo formado por los centros mineros de Zaragoza, Cáceres y San Jerónimo del Monte, ocasionando una inestabilidad importante a la zona. Es improbable que a partir de esta fecha los propietarios mineros introdujeran mayor incertidumbre a la región con la importación de nuevos esclavos ${ }^{37}$.

Gráfica No. 3. Llegadas de oro a España y producción en la Nueva Granada (en gramos)

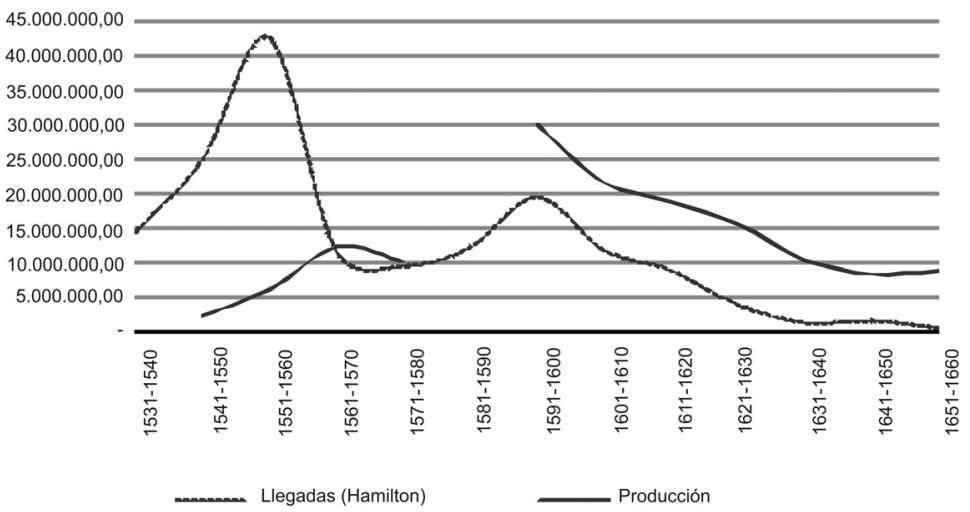

Fuente: Colmenares, Historia económica..., 332. ${ }^{38}$

Para 1633 se contabilizan en Zaragoza apenas 25 propietarios con 225 esclavos, donde una generación antes habían trescientos propietarios

\footnotetext{
36 Colmenares, Historia económica..., 304-305.

37 Cfr. Poveda, "Breve historia...", 210.

38 Colmenares no ofrece información de producción amonedada para la década de 1580. Por esta razón se presenta una discontinuidad en la gráfica, aunque las tendencias permanecen para los períodos siguientes.
} 
con cerca de tres mil esclavos y, para 1675, Juan Bueso Valdés reportaba que en toda Antioquia el número de esclavos no excedía los 400 y que en Zaragoza no quedaban sino 60. Además de la disminución que se registra por estas razones, la pérdida del control de la trata a partir de 1640, y el posterior encarecimiento del precio de los esclavos, fue un duro golpe a los propietarios de Cáceres, Remedios y Zaragoza. Sin embargo, es importante anotar que muchos de estos esclavos fueron desplazados a la frontera chocoana y otros entraron como libres a los circuitos económicos de la provincia.

Bajo estas circunstancias es dificil cuantificar el efecto del contrabando de hombres negros y mercancías a la Nueva Granada. Las cifras que utiliza Colmenares para comparar los registros de llegadas de oro a España elaboradas por Hamilton y las de producción pueden servir como indicador de lo que estaba pasando en la economía minera. Estas diferencias se pueden deber a la incapacidad de asegurar el comercio colonial y, por lo tanto, representan las fugas de los circuitos legales que se podían presentar tanto en las ferias como por el contrabando. ${ }^{39}$

Es importante anotar que, por una parte, las cifras de Hamilton han sido ampliamente discutidas, pues consideran que las 'cantidades absolutas' de oro llegadas a Sevilla, es decir, la adición de los quintos reales y de los envíos de los particulares representados por los pagos a comerciantes y por los envíos de los particulares, corresponden a la producción de oro de la Nueva Granada, lo que evidentemente no es así; y, por otra parte, las cifras de producción ofrecidas por Colmenares sólo tienen en cuenta el oro acuñado en moneda en Santafé. Sin embargo, los datos contenidos en la Gráfica No. 3 sirven como indicadores de los fenómenos que se han discutido. Se pueden observar en la gráfica No. 3 dos momentos importantes: en el primero, entre 1530 y 1580, las cifras de llegadas a España son superiores a las de la producción, y en el segundo, entre 1590 y 1600 , ocurre exactamente lo contrario.

Las dos tendencias son claramente diferenciables y continuas y, si sólo fueran divergencias periódicas que se compensaran en los siguientes períodos, esto se podría explicar mediante rezagos temporales entre la amonedación y la llegada del oro a España. Una posible explicación para la divergencia entre estas cifras, que teóricamente deberían ser

39 Colmenares, Historia económica..., 333. 
iguales, podría hallarse en la acumulación de capitales al interior de la Nueva Granada, que no salían a circuito externo y que comenzaban a consolidar una base diversificada de inversiones que mantenían en funcionamiento las economías regionales y locales. Otra posible explicación se podría encontrar en la incapacidad del sistema de flotas para asegurarse el comercio colonial, situación que le impedía a la metrópoli controlar toda la circulación aurífera que los comerciantes generaban en las ferias y, finalmente, una parte importante de este oro salía de la dinámica imperial por el contrabando.

En todo caso, ambas posibilidades reducían el monto total de oro que llegaba a España y, si se tienen en cuenta informaciones referentes al fraude permanente a las Cajas Reales, el contrabando ejercía una presión considerable sobre el fisco. Por ejemplo, para la segunda mitad del siglo XVII, el fiscal de la Audiencia, Fernando Prado, sostenía que dicho fraude habría alcanzado el $80 \%$ y, lugares como Zaragoza, sacaban cerca de 60.000 pesos anuales mientras registraban 12 a 14.000 pesos anuales. Situaciones similares debieron presentarse en los demás centros mineros de la zona, aunque, como se vio, las cifras de producción también se veían distorsionadas por el comercio y no sólo por el contrabando. ${ }^{40}$

Es plausible pensar que el fraude en los quintos reales comenzó a disminuir a partir de 1627, cuando se estableció la Casa de la Moneda de Santafé de Bogotá, pues toda persona podía declarar el oro allí sin perjuicio legal. La afluencia de oro a esta Casa de Moneda se vio además favorecida por el hecho que sólo se cobraba un 5\% de los derechos reales, mientras en otras regiones el impuesto se mantenía entre el quinto y el quinceavo y los derechos de fundición del $2.5 \%$, o cobos, quedaban eliminados. ${ }^{41}$

Sin embargo, la política fiscal se endureció a partir de 1664, cuando se comenzó a elevar de nuevo el impuesto del 5\% al 7,5\% a alrededor del 20\% para 1696. Aunque las cifras de producción muestran un incremento en la producción, esta alza en las tarifas seguramente incentivó el fraude en la fundición del oro y posiblemente la Casa de la Moneda de Santafé dejó de percibir el oro no declarado en las Cajas

\footnotetext{
40 Colmenares, Historia económica..., 335. Cfr. López, Migración y cambio..., 38 y 39.

41 Poveda, "Breve historia...", 210. Colmenares, Historia económica..., 336.
} 
Reales que tradicionalmente llegaba allí buscando las tarifas de impuestos más bajas. ${ }^{42}$

Como se vio en este apartado, aunque se esperaba que todo el producto de la minería llegara por diferentes vías a las fundiciones y pagara los impuestos, la evasión fiscal fue una constante en la colonia. La posibilidad de realizar operaciones comerciales, legales o ilegales, utilizando el oro en polvo como medio de pago y sin generar documentación oficial fomentaba una desviación de los registros de producción y una fuga de capitales a circuitos no controlados por la Corona. El monto de esta desviación es dificil de cuantificar, pero el contrabando era considerado como un delito grave y permanente por las autoridades y, a pesar de las reformas fiscales que buscaban disminuir el nivel de este, el éxito de las medidas no fue el esperado. ${ }^{43}$

Si se tiene en cuenta, además, el carácter de frontera relativamente móvil de la minería de aluvión; los privilegios legales que poseían los mineros, que hacía difícil cualquier proceso fiscal en su contra; el creciente control del oro en polvo como medio de pago por parte de los comerciantes; el peso del contrabando sobre la economía colonial y la existencia de centros mineros poco estudiados por la historiografia tradicional, el panorama minero del siglo XVII, que ha sido considerado como un interregno estéril entre los dos ciclos del oro, es diferente. Lo que se encuentra, posiblemente, es la coexistencia de ciclos e interciclos en la producción, que se superponen entre sí, y que generaron dinámicas de ampliación de las zonas incorporadas a la actividad económica.

De esta manera, el establecimiento y consolidación de nuevas zonas, como el valle de Aburrá, no obedece a la explicación tradicional que ha sostenido que se debía a una forma de supervivencia ante la crisis minera, sino que, por el contrario, obedece a una reconfiguración de la actividad en la región a través de nuevos ejes de explotación, que estaban dinamizando por vías diferentes la economía regional y que se veía expresada a través de procesos de territorialización exitosos que generaron nuevos centros primarios de intercambio comercial y de circulación de hombres y mercancías.

\footnotetext{
42 Colmenares, Historia económica..., 336.

43 Suárez, Oro y sociedad..., 258 y 259.
} 


\section{Referencias bibliográficas}

\section{Fuentes de Archivo}

Archivo Histórico de Antioquia, Colonia, Indios, tomo 24, documento 726, ff. $265 \mathrm{r}$ a $267 \mathrm{v}, 273 \mathrm{r}$.

. Colonia, Minas, tomo 110, Sección Santa Rosa, tomos 1, 2 y 3.

Colonia, Mortuorias, tomo 246, documento 5441, ff. 187-189.

Colonia, Real Hacienda, tomo 103, documento 2827, ff. 212r.

Colonia, Reales Provisiones, tomo 14, documento 486, ff. 15.

. Colonia, Tierras, tomo 183, documento 4610, ff. 1r a 2r.

Colonia, Tierras, tomo 183, documento 4611, ff. $3 \mathrm{r}$ a $6 \mathrm{r}$.

Colonia, Tierras, tomo 189, documento 4728, ff. 1r 1 r.

Escribanos, Juan José Lotero, ff. 56v-57r.

República, Estadística y censos, tomo 2689, documento 7, ff. 259r a $261 \mathrm{v}$.

\section{Fuentes Secundarias}

ALVAREZ,Víctor. La sociedad colonial, 1580-1720. En Jorge Orlando Melo (Dir). Historia de Antioquia. Medellín: Ed. Folio, 1988.

BREW, Roger. El desarrollo económico de Antioquia desde la Independencia hasta 1920.2 ed. Medellín: Universidad de Antioquia, 2000.

COLMENARES, Germán. La formación de la economía colonial (1500-1740). En: José A. Ocampo (comp.). Historia económica de Colombia. 4 ed. Bogotá:Tercer MundoFedesarrollo, 1994.

. Historia económica y social de Colombia, 1537-1719. 5 ed. Bogotá:Tercer Mundo, 1997.

JARAMILLO, Jaime. La economía del Virreinato (1740-1810). En José Antonio Ocampo (Comp.). Historia económica de Colombia. 4 ed. Bogotá:Tercer Mundo-Fedesarrollo, 1994.

LÓPEZ, Álvaro. Migración y cambio social en Antioquia durante el siglo XIX. Bogotá: Centro de Estudios Sobre Desarrollo Económico (CEDE), 1976.

MCFARLANE, Anthony. Colombia antes de la Independencia: Economía, sociedad y política bajo el régimen Borbón. Bogotá: Banco de la República-El Ancora, 1997.

NISSER, Pedro. La Minería en la Nueva Granada Bogotá: Banco de la República, 1990 (1834), 17.

PARSONS, James. La colonización antioqueña en el occidente de Colombia. 4 ed. Bogotá: Banco de la República-El Ancora, 1997.

POVEDA, Gabriel. Dos siglos de historia económica de Antioquia. Medellín: Pro Antioquia, 1979.

Breve historia de la minería. En Jorge Orlando Melo (Dir). Historia de Antioquia. Medellín: Ed. Folio, 1988. 
SUÁREZ, Ivonne. Oro y sociedad colonial en Antioquia,1575-1700. Medellín: Imprenta Departamental, 1993, 27.

TWINAM, Ann. Mineros, comerciantes y labradores: Las raíces del espíritu empresarial en Antioquia, 1763-1810. Medellín: FAES, 1985.

URIBE, María Teresa y ÁLVAREZ, Jesús. Raíces del poder regional: el caso antioqueño. Medellín: Universidad de Antioquia, 1988.

WEST, Robert C. Colonial placer mining in Colombia. Baton Rouge: Louisiana State University Press, 1952.

WILLIAMS, Caroline. "Resistance and rebellion on the Spanish frontier: native responses to colonization in the Colombian Choco, 1670-1690". Hispanic American Historical Review, 79.3, 1999:397-424. 